\title{
Management of secondary aortoesophageal fistula without graft extraction
}

\author{
Kazuya Kobayashi, MD, ${ }^{\mathrm{a}}$ Toshihiro Ohata, MD, PhD, ${ }^{\mathrm{a}}$ Hideki Ueda, MD, $\mathrm{PhD},{ }^{\mathrm{a}}$ and \\ Toshiaki Shichinohe, MD, PhD, ${ }^{\mathrm{b}}$ Sapporo, Japan
}

Aortoesophageal fistula is a rare but fatal complication of thoracic aortic surgery. The prognosis for the subtype of esophagoparaprosthetic fistula (EPPF), in which the implanted aortic prosthesis protrudes into the esophageal lumen, is especially poor. Here we report a case of EPPF that was successfully managed without removal of the aortic prosthesis.

\section{CLINICAL SUMMARY}

A 68-year-old woman was referred to our hospital for persistent slight fever and severe cough. Four years before, she had undergone total aortic arch replacement with elephant trunk for acute type A aortic dissection (Figure 1, A). At admission, a computed tomographic

\footnotetext{
From the Department of Cardiovascular Surgery, ${ }^{a}$ KKR Sapporo Medical Center, and the Division of Cancer Medicine, ${ }^{\mathrm{b}}$ Department of Surgical Oncology, Hokkaido University Graduate School of Medicine, Sapporo, Japan.

Disclosures: Authors have nothing to disclose with regard to commercial support.

Received for publication July 10, 2012; revisions received Aug 14, 2012; accepted for publication Sept 13, 2012; available ahead of print Oct 10, 2012.

Address for reprints: Kazuya Kobayashi, MD, Department of Cardiovascular Surgery, KKR Sapporo Medical Center, Hiragishi 1-6, Toyohira-ku, Sapporo, 062-0931,

Japan (E-mail: kakobayashi_tky@me.com).

J Thorac Cardiovasc Surg 2013; 145:e5-6

$0022-5223 / \$ 36.00$

Copyright (c) 2013 by The American Association for Thoracic Surgery

http://dx.doi.org/10.1016/j.jtcvs.2012.09.033
}

scan showed air entrapment along the polytetrafluoroethylene felt wrapping on the aortoprosthetic anastomosis (Figure 1, B). Mediastinitis was diagnosed, and antibiotic therapy was started. Endoscopy to investigate anemia (hemoglobin of $7.9 \mathrm{~g} / \mathrm{dL}$ ) revealed an esophageal ulcer $24 \mathrm{~cm}$ from the incisors, and the polytetrafluoroethylene felt wrapping was visible at the bottom of ulcer (Figure 2), confirming the diagnosis of EPPF.

A moderate inflammatory reaction was present without other signs of sepsis, blood culture results were negative, and follow-up computed tomographic scans showed no abscess formation or phlegmonous change of the soft tissue around the EPPF. Because infection was minimal, we decided to remove the part of the esophagus affected by the EPPF to avoid spreading contamination. The potentially infectious aortic graft was not resected, because an additional graft could be interposed if the infection could not be controlled postoperatively.

Simultaneous video-assisted thoracoscopic esophagectomy and esophageal reconstruction with stomach conduit were performed through the right posterior mediastinal route. There was no inflammation or purulence between the esophagus and the aortic graft, indicating fairly localized mediastinitis, and the esophagus was easily dissected. The EPPF was about $1 \mathrm{~cm}$ in diameter.
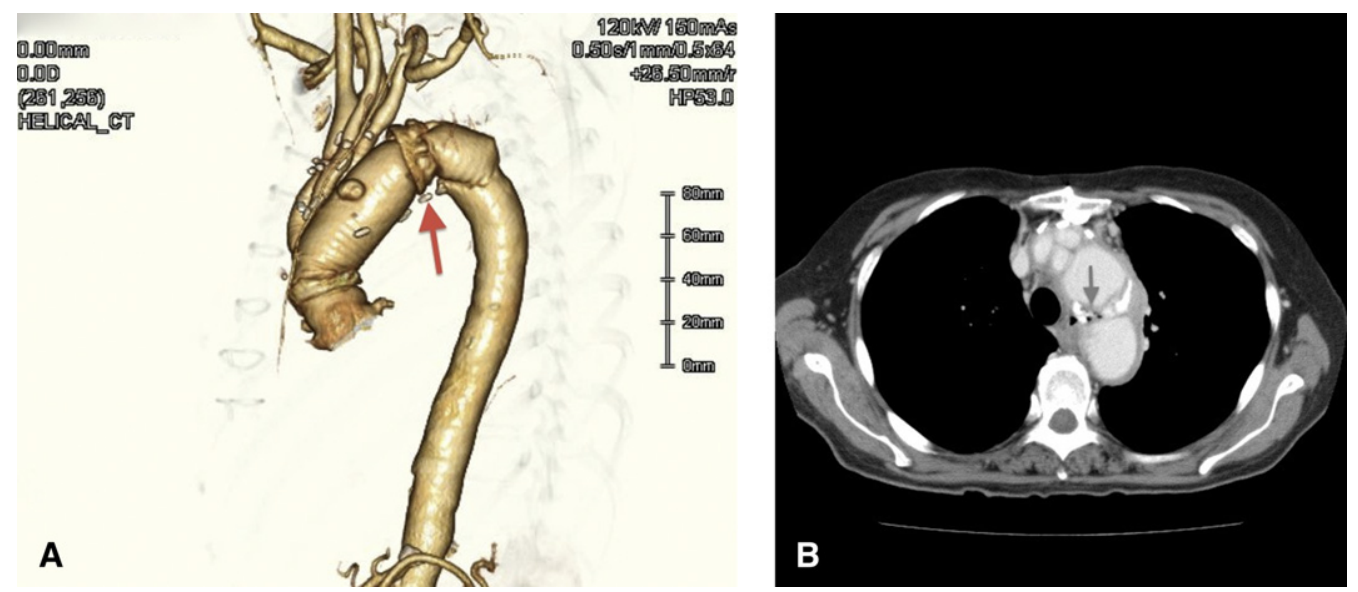

FIGURE 1. A, Three-dimensional reconstructed computed tomographic scan after total arch replacement with elephant trunk. Woven Dacron polyester fabric graft was used, and both proximal and distal anastomoses were covered with polytetrafluoroethylene felt from the outside. The elephant trunk was inserted into the distal anastomosis, extending $10 \mathrm{~cm}$ down to the descending aorta. The aortoesophageal fistula occurred at the distal anastomosis (red arrow). B, Axial computed tomographic scan shows air entrapment along the polytetrafluoroethylene felt wrapping the distal anastomosis (gray arrow). 


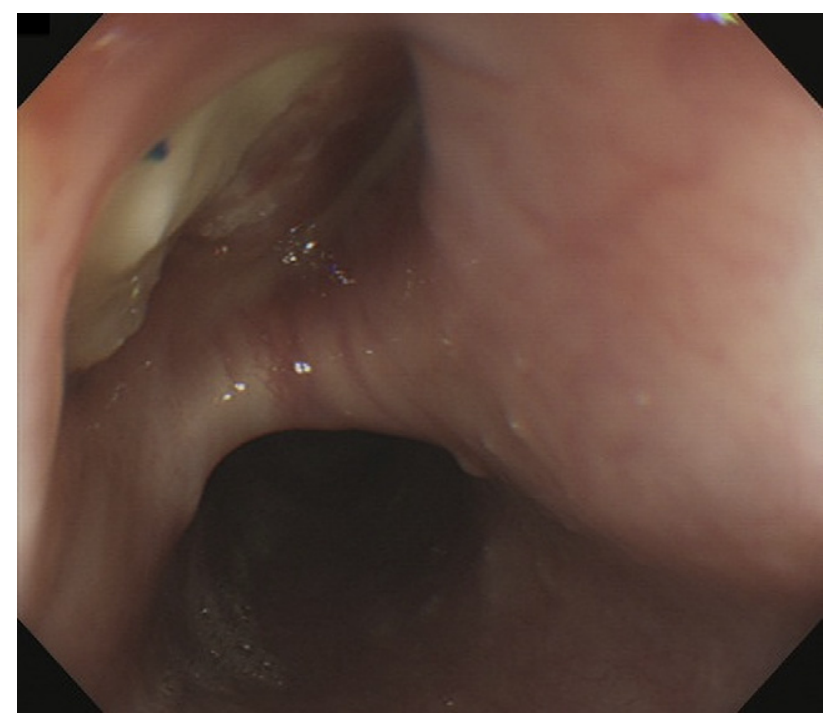

FIGURE 2. Endoscopic examination showed an esophageal ulcer $24 \mathrm{~cm}$ from the incisors, and the polytetrafluoroethylene felt wrapping on the distal anastomotic site was exposed at the bottom of diverticular shaped ulcer.

The surrounding esophageal tissue was rigidly adherent to the polytetrafluoroethylene strip, so it was cauterized, and the aortic prosthesis was covered with an omental flap.

On postoperative day 12 , the patient had hemorrhagic shock. A computed tomographic scan revealed massive bleeding at the posterior mediastinum and in the right pleural cavity. Emergency examination of the surgical site through a right thoracotomy could not reveal the source of the bleeding. The aortoprosthetic anastomosis was intact, with no evidence of disruption; however, the surface of the stomach conduit was necrotic and was therefore resected. A cervical esophagostomy and a feeding jejunostomy were created.

After the second operation, the patient recovered without further hemorrhage or sepsis. Intravenous antibiotics were stopped 7 weeks after the first operation. Five months after the first operation, esophageal continuity was reestablished with a free jejunal graft through a subcutaneous route. The patient recovered uneventfully from the third operation, and oral intake was resumed. At the 16-month follow-up examination, the patient was well and had no signs of mediastinitis on computed tomographic scans.

\section{DISCUSSION}

Aortoesophageal fistula is classified as primary (communication between the untreated diseased thoracic aorta and adjacent esophagus) or secondary (communication between the repaired aorta and esophagus). A secondary aortoesophageal fistula between the interposed prosthetic aortic graft and adjacent esophagus, as in our case, is known as EPPF. ${ }^{1}$ Typically, patients with EPPF are seen with sepsis and massive hematemesis, and the prognosis is extremely poor without treatment. Aggressive surgery involving esophageal resection, débridement, and aortic graft interposition or extra-anatomic bypass grafting offers the best likelihood of a cure ${ }^{2}$; however, both esophageal and aortic manipulations carry significant risk of mortality, especially in patients who have multiple comorbidities or are debilitated by sepsis or hemorrhage.

In our case, the infection was controlled for 16 months by esophageal manipulation alone, and a potentially infectious aortic graft was left in place. Graft extraction thus may not be indispensable for management of EPPF, and prompt removal of the perforated esophagus may be important for control of infection.

Until recent years, management of EPPF without aortic graft extraction was rarely successful. As reports of EPPF related to endovascular stent grafting increased, however, cases of successful management without removal of the prosthesis were also published. ${ }^{3}$ Although EPPF after endovascular stent grafting differs from EPPF after open aortic repair, these reports suggested the possibility of managing EPPF without removing the potentially infectious graft. Nevertheless, in most reports, prosthesis-preserving management had a fatal outcome. If the patient can physically tolerate an open aortic repair or has any sign of aortic graft infection, aggressive surgery to repair both the esophagus and the aortic graft is therefore still recommended. If, however, the patient's condition is so deteriorated as to preclude open aortic repair or there is no evidence of infection or hemorrhage, an esophagectomy before aortic repair ${ }^{4}$ or an esophagectomy alone may be acceptable for the management of EPPF.

\section{References}

1. Kawamoto S, Saiki Y, Oda K, Nitta Y, Akasaka J, Miyazaki S, et al. Successful management of esophagoparaprosthetic fistula after aortic surgery. Ann Thorac Surg. 2008;85:1449-51

2. Wickstrom PH, Streitz JM Jr, Erickson RV, Hoffman BD. Repair of aortoesophageal fistula after aortic grafting. Ann Thorac Surg. 1997;64:253-5.

3. Czerny M, Zimpfer D, Fleck T, Gotttardi R, Cejna M, Schoder M, et al. Successful treatment of an aortoesophageal fistula after emergency endovascular thoracic aortic stent-graft placement. Ann Thorac Surg. 2005;80: 1117-20.

4. Yamanaka K, Nonaka M, Iwakura A, Asao Y. Repair of aortoesophageal fistula after total aortic arch grafting. Interact Cardiovasc Thorac Surg. 2011;12: $655-6$ 